\title{
КЛИНИКО-РЕНТГЕНОЛОГИЧЕСКАЯ ХАРАКТЕРИСТИКА ПАЦИЕНТОВ С ЦЕРВИКАЛЬНЫМ СИНДРОМОМ ПОСЛЕ ТРАВМЫ НАДПЛЕЧЬЯ
}

\author{
Е. Б. Калинский ${ }^{1 凶}$, А. В. Черняев ${ }^{1}$, Л. Ю. Слиняков ${ }^{1}$, А. В. Лычагин ${ }^{1}$, Б. М. Калинский ${ }^{2}$, Ю. Р. Гончарук ${ }^{1}$
}

${ }^{1}$ Кафедра травматологии, ортопедии и хирургии катастроф, лечебный факультет

Первый Московский государственный медицинский университет им. И. М. Сеченова (Сеченовский университет), Москва

${ }^{2}$ Городская клиническая больница им. С. П. Боткина, Москва

\begin{abstract}
Состояние шейного отдела позвоночника (ШОП) находится во взаимосвязи с функциональным состоянием пояса верхней конечности. Повреждения пояса верхней конечности могут лежать в основе болевых ощущений в ШОП, в том числе вызывать цервикалгию. Целью исследования было оценить клинико-рентгенологическую картину ШОП у пациентов с цервикалгией, перенесших травму плечевого пояса. В исследование вошли 400 пациентов с жалобами на цервикалгию. Интенсивность боли оценивали по визуально-аналоговой шкале (VAS), ограничения жизнедеятельности - по опроснику NDI-RU (The Neck Disability Index, Russian language). При осмотре пациентов проводили оценку профиля позвоночника и локальных изменений в ШОП. Всем лицам, включенным в исследование, было выполнено рентгенологическое обследование ШОП в боковой и передне-задней проекциях, у 49,5\% пациентов проведена постуральная цифровая рентгенография позвоночника для оценки сагиттального профиля ШОП. Всем пациентам была сделана магнитно-резонансная томография (МРТ). По результатам комплексного клинико-лучевого обследования у пациентов были отмечены функциональные изменения, которые можно трактовать как причину развития цервикалгии. С точки зрения структурно-функционального диагноза определены различные статикодинамические нарушения. Таким образом, было выявлено, что болевой синдром в области ШОП является часто встречающейся проблемой у пациентов, перенесших травму надплечья.
\end{abstract}

Ключевые слова: цервикалгия, сагиттальный баланс, позвоночник, конус экономии, рентгенография, травма верхней конечности, шейный отдел позвоночника, общее строение

$\triangle$ Для корреспонденции: Калинский Евгений Борисович

ул. Трубецкая, д. 8, стр. 2, г. Москва, 119991; eugene_kalinsky@mail.ru

Статья получена: 15.03.2018 Статья принята к печати: 24.03.2018

DOI: $10.24075 /$ vrgmu.2018.017

\section{CLINICAL AND RADIOGRAPHIC CHARACTERISTICS OF PATIENTS WITH CERVICALGIA AFTER PREVIOUS INJURY TO THE PECTORAL GIRDLE}

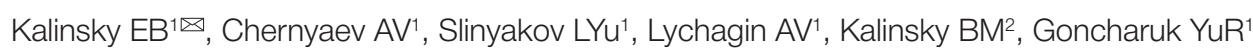

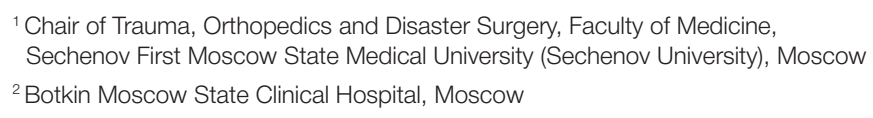

The health of the cervical spine (CS) and the functional state of the pectoral girdle are interdependent. Injuries to the pectoral girdle can be an underlying cause of CS pain, including cervicalgia. The aim of this study was to evaluate the condition of the cervical spine in patients with cervicalgia developed after a pectoral girdle injury using radiographic and physical examinations. The study included 400 patients complaining of cervicalgia. Pain intensity was evaluated on the visual analog scale (VAS); the impact of the condition on patients' lives was assessed using the Neck Disability Index (Russian language). During physical examinations, the general health of the spine was evaluated and abnormalities in the cervical spine were noted. All participants underwent a radiographic scan of the cervical spine in the lateral and anterior-posterior projections; $49.5 \%$ of patients underwent postural digital radiography to evaluate their CS sagittal profile. All patients received an MRI scan. Based on the results, we identified certain functional changes in the cervical spine which possibly caused cervicalgia. Structurally and functionally, the changes were divided into static and dynamic. We conclude that cervical spinal pain is a common problem among patients with previous pectoral girdle injury.

Keywords: cervicalgia, sagittal balance, spine, cone of economy, radiography, pectoral girdle injury, cervical spine, global alignment

$\triangle$ Correspondence should be addressed: Evgeny Kalinsky,

Trubetskaya 8, bl. 2, Moscow, 119991; eugene_kalinsky@mail.ru

Received: 15.03.2018 Accepted: 24.03.2018

DOI: 10.24075/brsmu.2018.017

В последнее десятилетие все большее значение уделяется структурно-функциональным нарушениям позвоночника как причине развития различных болевых синдромов не только аксиальной, но и периферической локализации. С биомеханической точки зрения позвоночник и пояс верхних и нижних конечностей можно рассматривать как сложную «вантовую» систему [1, 2]: позвоночник, занимая центральное положение, играет роль мачты, которая уравновешивается сбалансированной работой вант (плечевой пояс, таз, мышцы спины и конечностей). Изменение пространственной ориентации любого из звеньев этой биомеханической системы приводит к изменениям в положении и функционировании других частей. Цель адаптационных изменений - обеспечение эргономичной работы, т. е. сохранение постурального баланса туловища в пределах «конуса экономии» по 
Dubousset (рис. 1) [3-6]. Таким образом, функциональные состояния шейного отдела позвоночника (ШОП) и пояса верхней конечности следует рассматривать как взаимосвязанные [1, 3, 7, 8].

Повреждения пояса верхней конечности довольно распространены и в современной травматологической практике встречаются примерно в 15\% всех случаев возникновения скелетных травм [9]. Наиболее часто они возникают у пациентов молодого трудоспособного возраста в результате бытовых, спортивных, дорожнотранспортных травм.

Анализ результатов лечения лиц, поступивших с травмой плечевого пояса, позволяет выделить группу пациентов с синдромом цервикалгии. Данная группа гетерогенна и включает в себя пациентов разного возраста, проходивших как консервативное, так и оперативное лечение. Особый интерес представляют пациенты молодого и среднего возраста, не имевшие клинической картины цервикалгии до получения травмы. Развитие цервикального болевого синдрома приводит к формированию функциональных ограничений, снижает скорость и интенсивность реабилитации, ухудшает качество жизни пациентов [7]. Стоит отметить, что в настоящий момент в литературе отсутствуют статистически достоверные данные как о распространенности этого синдрома у пациентов с последствиями травмы плечевого пояса, так и о причинах его развития.

Целью исследования была оценка клиникорентгенологической картины ШОП у пациентов с цервикалгией, перенесших травмы плечевого пояса.

\section{ПАЦИЕНТЫ И МЕТОДЫ}

В исследование включено 400 пациентов, проходивших лечение на клинической базе травматологических отделений ГКБ им. С. П. Боткина кафедры травматологии, ортопедии и хирургии катастроф Первого МГМУ им. И. М. Сеченова в период с 2015 по 2018 г. Критерии включения: локальная боль в шее (клиническая картина цервикалгии) у пациентов мужского и женского пола;

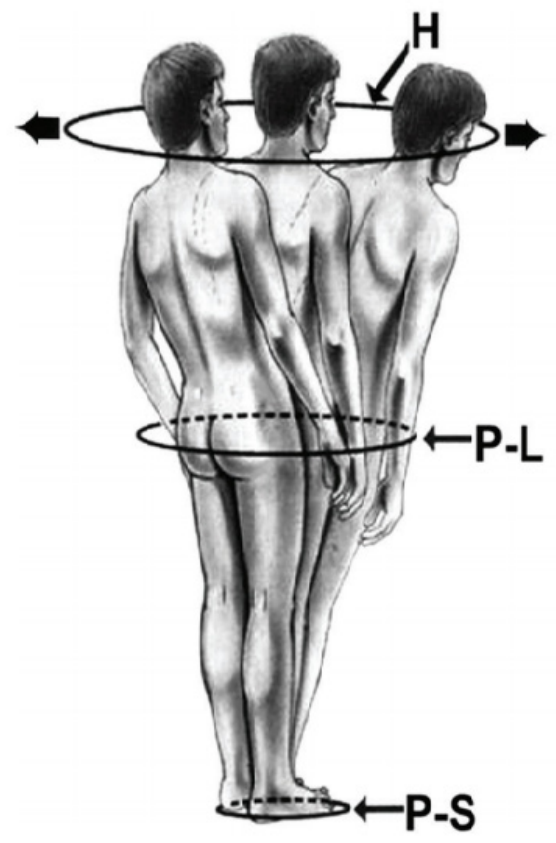

Рис. 1. Схема оптимальной позы человека с позиции концепции «конуса экономии" [3] возраст от 18 до 59 лет (средний возраст 41,3 \pm 1,1); изолированная травма одного надплечья в анамнезе (не менее 6 недель с момента получения).

Критерии исключения: клиническая картина цервикобрахиалгии с радикулярным синдромом; травма любого отдела позвоночника в анамнезе, стеноз спинномозгового канала ШОП различной этиологии; сочетанная травма.

Распределение пациентов по полу и возрасту представлено в табл. 1.

Хирургическое лечение пациентов было выполнено в 276 случаях (69\%), консервативное - в 124 (31\%).

Всем пациентам проведена клиническая оценка ортопедического статуса. Интенсивность боли оценивали по визуально-аналоговой шкале (VAS) [10, 11].

Оценку ограничения жизнедеятельности проводили по опроснику NDI-RU [12-14].

Рентгенографию ШОП выполняли в стандартных передне-задней и боковой проекциях (100\%). Функциональную рентгенографию ШОП не проводили в связи с низкой информативностью, обусловленной наличием болевого синдрома у пациентов и невозможностью проведения исследования хотя бы в относительно идентичных условиях.

Для оценки сагиттального профиля ШОП в 198 случаях (49,5\%) провели постуральную цифровую рентгенографию позвоночника в боковой и передне-задней проекциях [5]. Исследование позволяет оценить не только шейный баланс, но и так называемый global alignment (рис. 2) $[7,15-20]$.

Для оценки состояния межпозвонковых дисков, исключения стеноза спинномозгового канала ШОП всем пациентам проводили МРТ по стандартному протоколу Pfirrmann (в режимах T1, T2, STIR на томограсрах закрытого контура 1,5 Тесла) [21].

\section{РЕЗУЛЬТАТЫ ИССЛЕДОВАНИЯ}

Bce пациенты предъявляли жалобы на боли в шее. Интенсивность боли по VAS имела следующие значения: легкая боль - 18 пациентов (4,5\%), умеренная боль - 312 пациентов (78\%), сильная и нестерпимая боль 70 пациентов (17,5\%). Среднее значение интенсивности боли по VAS составило 5,6 \pm 0,45 балла.

По результатам анкетирования с применением опросника NDI-RU легкие нарушения жизнедеятельности выявлены у 85 пациентов (21,3\%), умеренные - у 290 (72,5\%), сильные - у 25 (6,2\%).

При осмотре пациентов проводили оценку профиля позвоночника в целом и локальных изменений в ШОП, в частности, а также объема движений ШОП (табл. 2).

При анализе рентгенограмм выявлены следующие локальные симптомы (табл. 3).

Деформации оси ШОП во фронтальной плоскости выявлено не было.

Результаты постуральной цифровой рентгенографии при оценке сагиттального баланса шеи представлены в табл. 4.

Таблица 1. Распределение по полу и возрасту

\begin{tabular}{|l|c|c|}
\hline & Мужчины & Женщины \\
\hline $18-44$ & $125(31,3 \%)$ & $101(25,2 \%)$ \\
\hline $45-59$ & $88(22 \%)$ & $86(21,5 \%)$ \\
\hline Всего & $213(53,3 \%)$ & $187(46,7 \%)$ \\
\hline
\end{tabular}




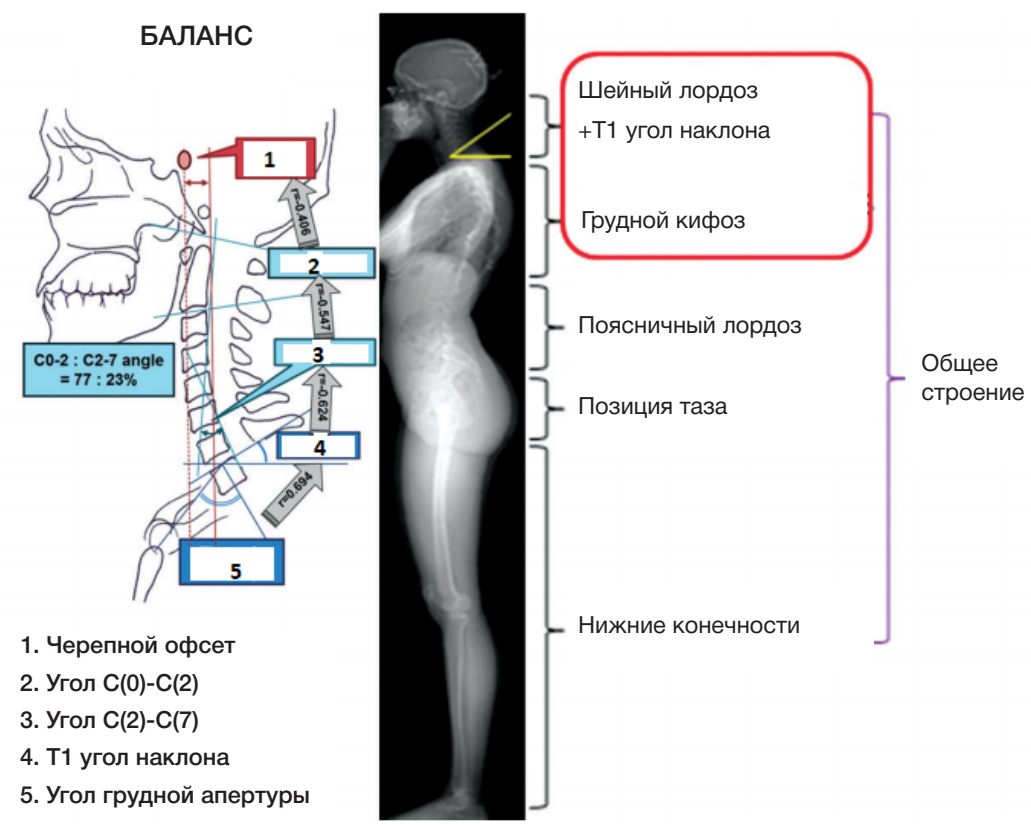

Рис. 2. Рентгенологические параметры сагиттального баланса [15, 19, 20]

Таблица 2. Результаты клинического осмотра пациентов

\begin{tabular}{|c|c|}
\hline Симптом & Количество пациентов \\
\hline \multicolumn{2}{|c|}{ Оценка профиля позвоночника } \\
\hline Плоская спина (сглаженность шейного и поясничного лордозов, грудного кифоза) & $98(24,5 \%)$ \\
\hline Круглая спина (усиленный грудной кифоз) & $47(11,7 \%)$ \\
\hline Плоско-вогнутая спина (усиленный грудной кифоз и поясничный лордоз) & $29(7,3 \%)$ \\
\hline Грудо-поясничный сколиоз & $247(61,8 \%)$ \\
\hline Перекос таза & $198(49,5 \%)$ \\
\hline \multicolumn{2}{|c|}{ Локальные изменения ШОП } \\
\hline Усиленный шейный лордоз & $186(46,5 \%)$ \\
\hline Сглаженный шейный лордоз & $199(49,8 \%)$ \\
\hline Повышенный тонус паравертебральных мышц & $359(89,3 \%)$ \\
\hline Ограничение флексии & $373(93,3 \%)$ \\
\hline Ограничение экстензии & $340(85 \%)$ \\
\hline Ограничение ротации & $381(95,3 \%)$ \\
\hline Ограничение боковой флексии & $391(97,8 \%)$ \\
\hline
\end{tabular}

Таблица 3. Результаты рентгенографического исследования

\begin{tabular}{|c|c|}
\hline Рентгенологический симптом & Количество (\%) \\
\hline Снижение высоты межпозвоночных дисков (относительное сравнение с дисками смежных сегментов) & $49(12,3 \%)$ \\
\hline «Лестничная» нестабильность тел позвонков (нарушение непрерывности линии, проведенной по задней стенке тел позвонков) & $273(68,3 \%)$ \\
\hline Артроз дугоотростчатых суставов & $23(5,8 \%)$ \\
\hline Локальное отклонение остистого отростка & $379(94,8 \%)$ \\
\hline Инклинация суставных отростков (смещение суставных поверхностей дугоотростчатых суставов с нарушением линии Хедли) & $367(91,8 \%)$ \\
\hline Спондилез & $12(3 \%)$ \\
\hline
\end{tabular}

Послепроведения МРТ увсехпациентов были выявлены признаки дегенеративных изменений межпозвонковых дисков ШОП I и II типов по Pfirmann [21], дегенеративных изменений тел позвонков не обнаружено. Признаки стеноза спинномозгового канала в исследуемой группе пациентов отсутствовали.

\section{ОБСУЖДЕНИЕ РЕЗУЛЬТАТОВ}

В исследование было включено 400 пациентов, перенесших травму надплечья, с клинической картиной цервикального болевого синдрома, развившегося в посттравматическом периоде. Клиническая картина во всех случаях складывалась из локального болевого синдрома, миофасциального синдрома, ограничения подвижности в ШОП. Рентгенологическое исследование не выявило у пациентов грубые дегенеративнодистросические изменения. При проведении в 198 случаях (49,5\%) оценки сагиттального баланса ШОП не было выявлено грубых биомеханических нарушений, что свидетельствует о локальных причинах болевого синдрома. Данные МРТ свидетельствуют о наличии 
Таблица 4. Параметры шейного баланса

\begin{tabular}{|l|c|c|}
\hline \multicolumn{1}{|c|}{ Параметр } & Полученное значение & Усредненный показатель [1, 15, 18] \\
\hline Угол С0-С2 & $-29^{\circ} \pm 1,3^{\circ}$ & $-30^{\circ}$ \\
\hline Угол C2-C7 & $-9,8^{\circ} \pm 0,9^{\circ}$ & $40^{\circ}$ \\
\hline T1S (T1 slope - угол наклона T1 позвонка) & $38,9^{\circ} \pm 1,2^{\circ}$ & $4 \mathrm{~cm}$ \\
\hline C2-C7 SVA (сагиттальная вертикальная ось) & $3,9 \pm 0,5 \mathrm{~cm}$ & $44^{\circ}$ \\
\hline TIA (thoracic inlet angle - угол входа в грудную клетку) & $43^{\circ} \pm 1,4^{\circ}$ & \\
\hline
\end{tabular}

у пациентов начальных стадий дегенеративнодистрофических изменений позвоночно-двигательных сегментов без признаков диск-радикулярных конфликтов и стенозирования спинномозгового и латеральных каналов. Таким образом, выявленные у пациентов изменения не относятся к структурным и носят функциональный характер. С точки зрения функционального диагноза определяются различные статико-динамические нарушения (гипермобильность, гипомобильность позвоночно-двигательных сегментов), что в целом можно трактовать как дислокационный функциональный синдром в рамках фасет-синдрома (синдром дугоотростчатых суставов, facet joint syndrome) [22, 23].

В зависимости от выявленных статико-динамических изменений пациентам можно рекомендовать соответствующий план реабилитационного лечения с целью купирования цервикального болевого синдрома.
Однако необходимо дальнейшее исследование для определения степени корреляции выявленных изменений с различными видами повреждений надплечья и типов их хирургического лечения, а также другими структурнофункциональными нарушениями позвоночника и таза.

\section{ВЫВОДЫ}

Болевой синдром в области ШОП является часто встречающейся проблемой у пациентов, перенесших травму надплечья. Повреждения надплечья могут приводить к развитию функциональных нарушений ШОП, являющихся причиной цервикалгии. Дальнейшее изучение функциональных нарушений ШОП у пациентов, перенесших травмы надплечья, позволит разработать методы просрилактики этих нарушений и сформировать схемы восстановительного лечения.

\section{Литература}

1. Капанджи А. И. Позвоночник. Физиология суставов. 6 изд. М.: Изд-во «Эксмо»; 2009. 327 с.

2. Dubousset J, Challier V, Farcy JP, Schwab FJ, Lafage V. Spinal alignment versus spinal balance. In: Haid RW, Schwab FJ, Shaffrey Cl, Youssef JA (ed.)/. Global Spinal Alignment: Principles, Pathologies, and Procedures St. Louis, MO: Quality Medical Publishing, 2014. p. 3-9.

3. Dubousset J. Three-dimensional analysis of the scoliotic deformity. In: The pediatric spine: principles and practice. New York: Raven Press; 1994: 479-496.

4. Hasegawa K, Okamoto M, Hatsushikano S, Shimoda H, Ono M, Watanabe K. Normative values of spino-pelvic sagittal alignment, balance, age, and health-related quality of life in a cohort of healthy adult subjects. Eur Spine J. 2016; 25 (11): 3675-86. https://doi. org/10.1007/s00586-016-4702-2

5. Mac-Thiong JM, Roussouly P, Berthonnaud E, Guigui P. Sagittal parameters of global spinal balance: normative values from a prospective cohort of seven hundred nine Caucasian asymptomatic adults. Spine. 2010; 35 (22): E1193-E1198. https://doi.org/10.1097/brs.0b013e3181e50808

6. Ames CP, Smith JS, Scheer JK, Bess S, Bederman SS, Deviren V et al. Shaffrey Impact of spinopelvic alignment on decision making in deformity surgery in adults: A review. J Neurosurg Spine. 2012; 16 (6): 547-64. https://doi: 10.3171/2012.2.SPINE11320

7. Бурцев А. В., Рябых С. О., Котельников А. О., Губин А. В. Клинические аспекты сагиттального баланса у взрослых. Гений ортопедии. 2017; 23 (2): 228-35. https://doi. org/10.18019/1028-4427-2017-23-2-228-235

8. Калинский Е. Б., Калинский Б. М., ГончарукЮ. Р., Усманова Н. Х. Подходы к лечению повреждений акромиально-ключичного сустава III типа (по Rockwood). Мануальная терапия. 2017; 66 (2): 65-68.

9. Котельников Г. П., Миронов С. П., редакторы. Травматология. Национальное руководство. М.: ГЭОТАР-Медиа; 2008: 808 с.

10. Scott J, Huskisson EC. Vertical or horizontal visual analogue scales. Ann Rheum Dis. 1979; 38: 560.

11. Gould D. et al. Visual Analogue Scale (VAS). Journal of Clinical

Nursing 2001; 10: 697-706

12. Vernon H, Mior, S. (1991). The Neck Disability Index: A study of reliability and validity. Journal of Manipulative and Physiological Therapeutics. 14 (7): 409-15.

13. Bakhtadze MA, Vernon $\mathrm{H}$, Zakharova OB, Kuzminov $\mathrm{KO}$, Bolotov DA. The Neck Disability Index-Russian Language Version (NDI-RU): A Study of Validity and Reliability. Spine (Phila Pa 1976). 2015 Jul 15; 40 (14): 1115-1. DOI: 10.1097/ BRS.0000000000000880.

14. Бывальцев В. А., Белых Е. Г., Алексеева Н. В., Сороковиков В. А. Применение шкал и анкет в обследовании пациентов C дегенеративным поражением поясничного отдела позвоночника: методические рекомендации. Иркутск: ФГБУ "НЦРВХ" СО РАМН, 2013. 32 c.

15. Scheer JK., Tang JA, Smith JS, Acosta Jr FL, Protopsaltis TS, Blondel B et al. Cervical spine alignment, sagittal deformity, and clinical implications: a review. J Neurosurg Spine. 2013; 19 (2): 141-159. https://doi.org/10.3171/2013.4.SPINE12838

16. Ozer AF, Kaner T, Bozdoğan C.. Sagittal Balance in the Spine. Turkish Neurosurgery. 2014; 24 (1): 13-19.

17. Le Huec JC, Demezon H, Aunoble S. Sagittal parameters of global cervical balance using EOS imaging: normative values from a prospective cohort of asymptomatic volunteers. Eur Spine J. 2015; 24 (1): 63-71. https://doi.org/10.1007/s00586-014-3632-0

18. Liu S, Schwab F, Smith JS, Klineberg E, Ames CP, Mundis G et al. Likelihood of reaching minimal clinically important difference in adult spinal deformity: a comparison of operative and nonoperative treatment. Ochsner J. 2014; 14 (1): 67-77.

19. Vialle R, Levassor N, Rillardon L, Templier A, Skalli W, Guigui P. Radiographic analysis of the sagittal alignment and balance of the spine in asymptomatic subjects. J Bone Joint Surg Am. 2005; 87 (2): 260-7.

20. Lafage V, Schwab F, Skalli W, Hawkinson N, Gagey PM, Ondra S et al. Standing balance and sagittal plane spinal deformity: analysis of spinopelvic and gravity line parameters. Spine. 2008; 33 (14): 1572-78. https://doi: 10.1097/BRS.0b013e31817886a2

21. Pfirrmann CW, Metzdorf A, Zanetti M, Hodler J, Boos N. 
Magnetic resonance classification of lumbar intervertebral disc degeneration. Spine (Phila Pa 1976). 2001 Sep 1; 26 (17): 1873-8.

22. Авоян Т. К. Консервативное лечение больных с синдромом межпозвонковых суставов при поясничном остеохондрозе [диссертация]. М.: 1998

23. Жандаров К. А. Стеноз межпозвоночных каналов шейного отдела позвоночника [диссертация]. М.: 2016.

Therapeutics. 14 (7): 409-15.

13. Bakhtadze MA, Vernon $\mathrm{H}$, Zakharova OB, Kuzminov $\mathrm{KO}$ Bolotov DA. The Neck Disability Index-Russian Language Version (NDI-RU): A Study of Validity and Reliability. Spine (Phila Pa 1976). 2015 Jul 15; 40 (14): 1115-1. DOI: 10.1097/ BRS.0000000000000880.

14. Bivaltsev VA, Belikh EG, Alekseeva NV, Sorokovikov VA. Primenenie shkal I anket $\mathrm{v}$ obsledovanii patsientov $\mathrm{S}$ degenerativnim porazheniem poyasnichnogo otdela pozvonochnika; metodicheskie rekomendatsii. Irkutsk: 2013. 32 s.

15. Scheer JK., Tang JA, Smith JS, Acosta Jr FL, Protopsaltis TS, Blondel B et al. Cervical spine alignment, sagittal deformity, and clinical implications: a review. J Neurosurg Spine. 2013; 19 (2): 141-159. https://doi.org/10.3171/2013.4.SPINE12838

16. Ozer AF, Kaner T, Bozdoğan Ç. Sagittal Balance in the Spine. Turkish Neurosurgery. 2014; 24 (1): 13-19.

17. Le Huec JC, Demezon H, Aunoble S. Sagittal parameters of global cervical balance using EOS imaging: normative values from a prospective cohort of asymptomatic volunteers. Eur Spine J. 2015; 24 (1): 63-71. https://doi.org/10.1007/s00586-014-3632-0

18. Liu S, Schwab F, Smith JS, Klineberg E, Ames CP, Mundis G et al. Likelihood of reaching minimal clinically important difference in adult spinal deformity: a comparison of operative and nonoperative treatment. Ochsner J. 2014; 14 (1): 67-77.

19. Vialle R, Levassor N, Rillardon L, Templier A, Skalli W, Guigui P. Radiographic analysis of the sagittal alignment and balance of the spine in asymptomatic subjects. J Bone Joint Surg Am. 2005; 87 (2): 260-7.

20. Lafage V, Schwab F, Skalli W, Hawkinson N, Gagey PM, Ondra S et al. Standing balance and sagittal plane spinal deformity: analysis of spinopelvic and gravity line parameters. Spine. 2008; 33 (14): 1572-78. https://doi: 10.1097/BRS.0b013e31817886a2

21. Pfirrmann CW, Metzdorf A, Zanetti M, Hodler J, Boos N. Magnetic resonance classification of lumbar intervertebral disc degeneration. Spine (Phila Pa 1976). 2001 Sep 1; 26 (17): 1873-8.

22. Avoyan TK. Konservativnoe lechenie bol'nih s sindromom mezhpozvonkovih sustavov pri poyasnichnom osteohondroze [dissertacija). M.: 1998.

23. Zhandarov KA. Stenoz mezhpozvonochnix kanalov sheinogo otdela pozvonochnika. [dissertacija]. M.: 2016. 